\title{
Desempeño de las doulas en la atención de la mujer y su familia durante el periodo gestacional, parto y post parto en Costa Rica $^{1}$
}

Beatriz Rodríguez Fernández ${ }^{2}$

Katherine Durán Castillo ${ }^{3}$

Institución: Sistema de Estudios de Posgrado. Universidad de Costa Rica

\section{COMO CITAR}

Rodríguez, B. y Durán, K. (2016). Desempeño de las doulas en la atención de la mujer y su familia durante el periodo gestacional, parto y post parto en Costa Rica. Rev. Enfermería Actual de Costa Rica, 30, 1-21. DOI: http://dx.doi.org/10.15517/revenf.v0i30.22112

\section{RESUMEN}

Introducción: La presente investigación representa un acercamiento al desempeño en la atención que brindan las doulas a la mujer embarazada y sus familias en Costa Rica en la cual se evidencia sus conocimientos respecto de sus intervenciones con las mujeres embarazadas y las familias.

Métodos: Se realizó una investigación cualitativa, con un enfoque en el modelo planteado por Maxwell, cuyos objetivos fueron concretados con el método etnográfico. Para recolectar la información, se aplicó entrevistas a profundidad y observaciones, entre otros, a un grupo de siete doulas que laboran en Costa Rica de forma independiente.

Resultado principal: todas las doulas en nuestro país se capacitan en prácticas alternativas para complementar su labor, así como prácticas e intervenciones que no forman parte del perfil "internacional”, ya que en Costa Rica no existe una entidad que regule dicha labor.

Conclusión: el apoyo continuo a la mujer y su familia durante todo su embarazo, parto y postparto por una persona calificada que respete los principios éticos de su práctica, permite mejorar la experiencia del parto, motivo por el que se debe sensibilizar a la población de enfermeros obstetras en cuanto a mejorar la calidad en la atención que se brinda a las mujeres embarazadas de modo que promueva el acompañamiento continuo.

Palabras clave. Acompañamiento, doulas, Enfermería-obstétrica, familia-gestante, parto-respetado.

\footnotetext{
${ }^{1}$ Fecha de recepción: 12 de agosto del 2015

Fecha de aceptación: 9 de octubre del 2015

${ }^{2}$ Enfermera Obstetra, Hospital La Católica, San José. Correo electrónico: betirf@hotmail.com

${ }^{3}$ Enfermera Obstetra, Hospital México, San José. Correo electrónico: kaduran06@gmail.com
} 


\section{Performance of doulas in the care of the woman and her family during the gestational period, delivery and postpartum in Costa Rica ${ }^{1}$}

Beatriz Rodríguez Fernández ${ }^{2}$

Katherine Durán Castillo ${ }^{3}$

Institution: Postgraduate Studies. University of Costa Rica

\section{CITED:}

Rodríguez, B. y Durán, K. (2016). Performance of doulas in the care of the woman and her family during the gestational period, delivery and postpartum in Costa Rica. Rev. Enfermería Actual de Costa Rica, 30, 1-21. DOI: http://dx.doi.org/10.15517/revenf.v0i30.22112

\section{ABSTRACT}

Introduction: This research represents an approach to performance in doulas care with pregnant women and their families in Costa Rica which is can demonstrate knowledge that possess them in their interventions with pregnant women and their families.

Methods: we conducted a qualitative research, with a focus on the model proposed by Maxwell. Using the ethnographic method for the achievement of the proposed objectives. For the collection of the information were conducted in-depth interviews and observations, among other things to a group of seven doulas working in Costa Rica independently.

Primary outcome: all doulas in our country are trained in alternative practices to complement their work, most of doulas conducted practices and interventions that do not form part of the "international" profile since we don't have an entity that regulates the work of doulas in Costa Rica.

Conclusions: continuous support to the woman and her family throughout her pregnancy, childbirth and postpartum by a qualified person and which respects the principles of ethical practice, improves the experience of childbirth and reduces the risks of an adverse event for the mother or the baby, this is why it's important to educate the population of nurse-midwives to improve the quality of care provided to expectant mothers and promote the continuous support.

Key words. Accompaniment, doulas, expectant-family, nurse-midwife, respected-childbirth.

\footnotetext{
${ }^{1}$ Date of acceptance: August 12, 2015

${ }^{2}$ OB Nurse, Hospital La Católica, San Jose. Email: betirf@hotmail.com

${ }^{3}$ OB Nurse, Hospital México, San José. Email: kaduran06@gmail.com
} 


\section{Revista Electrónica Enfermeria Actual en costa Rica}

\section{INTRODUCCIÓN}

En los últimos años se ha enfatizado la importancia de crear redes de apoyo que faciliten el proceso de embarazo y parto a las mujeres, deber a cargo del personal de salud, principalmente de los profesionales en enfermería obstétrica respecto de crear un ambiente adecuado que promueva el respeto, la intimidad, el empoderamiento de la mujer y su familia en la toma de decisiones y acorde a las necesidades de cada mujer a la hora del parto para así ayudar a disminuir los riesgos derivados de un mal manejo.

A partir de lo anterior cabe mencionar que la obstetricia ha influido en otras profesiones o prácticas, como por ejemplo en las doulas, mujeres capacitadas para dar acompañamiento, apoyo, y orientación durante el proceso de embarazo con base en conocimientos adquiridos de su experiencia a lo largo de los años.

Al respecto, Romero (2009) menciona que fue Dana Raphael, conocida antropóloga, la primera persona que empleó este término, el cual proviene de la antigua Grecia y que se refiere a mujeres experimentadas, encargadas de ayudar a las futuras madres en la crianza de sus hijos e hijas. Otros investigadores como Kennel, Klaus, McGrath, Robertson \& Hinkley (1991) realizaron una serie de estudios aleatorios controlados (p.2) y extendieron el empleo de este término a mujeres ayudantes tanto en el postparto como durante el parto.

El rol de la doula es proveer a la mujer embarazada una preparación adecuada para el parto, técnicas y estrategias que la guíen y le brinden apoyo, así como construir una relación de trabajo en equipo con el personal de enfermería, de modo que se promueva la comunicación entre la paciente y los profesionales de la salud. Entre algunas de las técnicas que utilizan se encuentra la imagenología, masaje, acu presión y patrones de respiración para reducir el dolor durante el parto (Papagni y Buckner 2006, p.12).

En nuestro país, representan un gremio poco conocido, sin embargo en la actualidad muchas mujeres y sus familias solicitan sus servicios en el ámbito privado, tema sobre el que Chinchilla (2010) se refiere en "Aliadas a la hora del parto" en donde indica que "una doula no es una médica, una enfermera ni una partera, sino una mujer que sirve de compañía y apoyo a la parturienta durante su proceso de labor o durante los días posteriores al nacimiento del bebé" (s.p.).

A lo largo de la historia se ha demostrado el acompañamiento de la doula a través de diversos estudios internacionales que demuestran los beneficios que implica su acompañamiento, entre los que Valdés y Morlans (2005) tal como reducción del tiempo del trabajo de parto, de la necesidad de anestesia o analgesia, así como de la incidencia de cesáreas; de igual forma, comentan que los partos naturales (vía vaginal, sin oxitocina, anestesia o fórceps) son más frecuentes en las mujeres que cuentan con la presencia de una doula.

En Brasil, Mendoza, Silva y Alves (2010), investigaron distintos aspectos referentes a la participación del acompañante en la atención del parto humanizado y su interfaz con el equipo de salud y concluyeron que se trata de un proceso en construcción que involucra aspectos físicos y ambientales de las instituciones de salud, de profesionales capacitados y de la propia cultura de las usuarias que siguen comportándose de manera muy sumisa en relación con los derechos que como ciudadanas poseen. 
Por otro lado, Uribe y Viveros (2009) mencionan que cuando las Doulas asisten a las mujeres durante el parto, son más cortos, presentan menos complicaciones, los bebés tienden a ser más sanos y la lactancia materna se inicia más fácilmente (p.103).

Debido a lo anterior, entre las razones primordiales para realizar esta investigación fue vivir el proceso de atención en Costa Rica que realizan las doulas a las mujeres en el periodo gestacional, parto y post parto, así como a sus familias, comprender el enfoque con el que brindan la atención y observar al mismo tiempo los efectos del acompañamiento que ellas brindan a la población. De la información presentada, surgen las siguientes preguntas de investigación: ¿Cómo y dónde se capacitan las doulas en Costa Rica para brindar la atención a la familia gestante? ¿Cómo se desempeñan las en la atención de la mujer en la gestación parto y post parto?, ¿Cuál es el enfoque de la atención que brindan en Costa Rica?

De las preguntas planteadas surgió el objetivo general, el cual consistió en analizar el desempeño de las doulas que laboran en Costa Rica en la atención de la mujer y su familia durante el periodo gestacional, parto y post parto.

\section{MATERIALES Y MÉTODOS}

Para la presente investigación, se seleccionó un grupo de siete doulas que laboran en Costa Rica de forma independiente, quienes son las informantes claves del estudio. Con respecto a la aproximación del objeto de estudio se estableció un vínculo entre las investigadoras y las doulas (objeto de estudio), con lo que se logró una aproximación epistemológica que trasciende del objeto al sujeto, es decir, como lo menciona Rojas (2008) las investigadoras lograron ir más allá, fueron distintas y distantes del sujeto, en este caso, de las doulas.

El tipo de estudio corresponde al cualitativo, puesto que según Martínez (2006), "la investigación cualitativa trata de identificar la naturaleza profunda de las realidades, su estructura dinámica, aquella que da razón plena de su comportamiento y manifestaciones" (p.128), mientras que para la consecución de los objetivos se empleó el método etnográfico.

Se trabajó con base en el diseño cualitativo que plantea Maxwell (2005), el cual es un modelo interactivo que consiste en los componentes de un estudio de investigación y en los caminos que estos componentes pueden afectar. Dicho modelo se detalla a continuación:

Propósito: El propósito de esta investigación fue indagar sobre aspectos que caracterizan a las doulas, mediante un análisis de su desempeño en cuanto a la atención del parto y demás interacciones que tienen con mujeres en periodo de gestación y sus familias, además de mitigar estigmas sobre este grupo para aumentar la comprensión por parte del personal de salud en su labor en la atención del parto. Por último, pero no menos importante, se buscaba mostrar a los profesionales en obstetricia otras formas de abordar el proceso de embarazo, parto y post parto, con el fin de mejorar la atención que reciben la madre, el neonato y sus familias.

Contexto conceptual: En este punto se establece qué sucede con el tema en estudio, las teorías, creencias y resultados de investigaciones previas que guiarán la investigación actual, además de experiencias personales, literatura y estudios preliminares que ayudan a la comprensión del grupo o personas en estudio. Debido a que se 
trabajó con el método etnográfico, las investigadoras no realizaron una búsqueda bibliográfica previa sobre el tema planteado, con el fin de no crear juicios o criterios sobre el tema antes de iniciar la investigación, por ende, dicha revisión se aplazó hasta que se obtuviera los resultados de la investigación con el fin de orientar su análisis.

Método: Para elaborar la presente investigación, se utilizó el método etnográfico, el cual, según Rodríguez, Gil y García (1999), es "el método de investigación por el que se aprende el modo de vida de una unidad social concreta", aparte de que "persigue la descripción o reconstrucción analítica de carácter interpretativo de la cultura, formas de vida y estructura social del grupo investigado" (p. 44).

Con el fin de alcanzar los objetivos planteados desde la metodología etnográfica, se desarrolló los siguientes puntos:

- Determinación del nivel de participación: hubo varias limitantes en la participación de las actividades y labores que desempeñan las doulas al momento del parto, debido a que ellas creen que los factores externos ajenos a la madre en el momento de la labor de parto y parto pueden influir negativamente en el adecuado curso del mismo, de modo que retrase el nacimiento del nuevo bebé o lo inhiba por completo, ya que es un momento en el que -ante todo- ellas velan por la privacidad de la madre, su tranquilidad y comodidad y evitan todos los factores que podrían representar un momento de estrés para ellas y su familia. Por lo anterior, a pesar de que se solicitó observar las intervenciones de las doulas durante el parto, ellas se negaron debido a sus fuertes convicciones y creencias como guardianas y acompañantes de la mujer en el momento del parto.

Debido a tales obstáculos, únicamente se logró asistir como observadoras participantes al Encuentro de la Red de Doulas de Latinoamérica y el Caribe, realizado el día 20 de octubre del 2013, donde participaron aproximadamente 14 tanto costarricenses como extranjeras, a lo que se agrega tres observaciones de las charlas de las doulas con las parejas en la fundación Previda, y nueve entrevistas a profundidad realizadas a siete que laboran en Costa Rica, con el fin de obtener la información necesaria para el cumplimiento de los objetivos de la investigación planteada

- Recolección de la información: se utilizó técnicas tales como entrevistas a profundidad estructuradas y no estructuradas, observaciones durante el periodo gestacional y notas de campo, principalmente de tipo condensadas, las cuales Valle (1999) define como notas "tomadas en el momento o inmediatamente después de una sesión de trabajo de campo" (p.170), así como de tipo expandidas cuyo objetivo es registrar con más detenimiento y detalle los datos obtenidos por las investigadoras en cada observación, para evitar la pérdida de información importante. De igual modo, se recopiló material de un documental llamado "Así nacemos" y varios videos de partos realizados en Costa Rica por Clea Eppelim recuperados de una página en internet llamada Vimeo, además del documental "El derecho a elegir" realizado y proporcionado por una de las doulas, elaborado también en Costa Rica. En estos documentales y videos se observa la intervención de las doulas con diferentes mujeres y sus familias en el momento de la labor de parto y postparto. Por último, se utilizó fotografías y charlas con el fin conseguir la información que mejor descubriera las estructuras significativas que dan razón de la conducta de las doulas. 
- Nivel de objetividad: se obtuvo un alto nivel de objetividad, debido al tipo de investigación realizada, ya que la de tipo etnográfico tiene un enfoque fenomenológico. Por otro lado, basado en el modelo de investigación etnográfica y nivel de objetividad que explica Martínez (1997), se evitó la subjetividad al descubrir las acciones de las doulas desde las diversas perspectivas que ellas mismas relatan, motivo por el que se adecuó las entrevistas a las categorías empíricas de ellas para así comprender y analizar el significado que tienen sus acciones y desempeño como doulas desde la objetividad de sus vivencias.

- Análisis de los datos: Con el fin de lograr un adecuado análisis de los resultados, se empleó la triangulación de los datos recolectados por las investigadoras, tomando en cuenta sus percepciones derivadas de las vivencias con el grupo investigado, sin olvidar la triangulación de las categorías y unidades de análisis establecidas a lo largo de la investigación.

- Generalización de los resultados: las investigadoras generalizaron toda información recolectada de las participantes, con el fin de obtener los resultados de la investigación y comprender la compleja estructura del desempeño de las doulas con la familia gestante.

Validez: Se elaboró una recolección exhaustiva de información para validar el estudio, a través de las técnicas e instrumentos como las notas de campo, las entrevistas a profundidad y las observaciones, lo cual se ajusta a las palabras de Martínez (2006), quien considera que las investigaciones etnográficas logran un alto nivel si estudian la realidad tal como es, de ahí el porqué de los métodos por utilizar.

\section{Unidades y categorías de análisis}

La primera unidad de análisis fue la socioeducativa a partir de la que se caracterizó la población de las doulas en Costa Rica, tomando en cuenta diferentes categorías como la edad, estado civil, nacionalidad, nivel educativo y lugar de formación, entre otras.

Otra unidad de análisis fue su desempeño en la atención de la mujer embarazada y sus familias, para lo que se empleó las diferentes etapas del embarazo: preparto, parto y postparto como categorías de análisis, mientras que la triangulación de los datos obtenidos fue por medio de las entrevistas, con las observaciones y la teoría encontrada.

\section{Salida del campo}

Después de aplicar las entrevistas y observaciones necesarias y llegar al punto de saturación de la información obtenida, se realizó la salida del campo de tal manera que en caso de que las investigadoras tuvieran la necesidad de volver a interaccionar con las doulas, o de aclarar la información obtenida en algún momento, no hubiese ningún inconveniente. 


\section{Consideraciones éticas}

Con respecto a las consideraciones éticas para el desarrollo de la investigación, se fundamentan en un componente moral; por ende, se consideró la importancia bioética en la investigación científica con personas, debido a los principios de responsabilidad profesional por parte de las investigadoras. Dado lo anterior, se trabajó desde el enfoque principialista de la bioética, por medio del que fue posible poner en práctica los principios bioéticos ante cada situación, tales como el principio de autonomía, beneficencia, no maleficencia y justicia además de la veracidad de la información, privacidad y divulgación de los resultados.

Se utilizó un consentimiento informado, el cual permitió tanto a las doulas participantes, como a las madres y sus parejas, tener el suficiente conocimiento y entendimiento de los objetivos de la investigación, así como la responsabilidad asumida por las investigadoras con el manejo de los datos obtenidos y los beneficios derivados de la investigación, entre otros aspectos.

\section{RESULTADOS}

\section{Caracterización de la población}

Se aplicó entrevistas a siete doulas de la GAM, seis son doulas de parto y una es de postparto, con edades entre los 32 y 56 años, tres casadas, dos divorciadas y dos en unión libre. Todas con la experiencia de ser madres con hijos de diferentes edades. Dos de ellas son de nacionalidad estadounidense, una guatemalteca y cuatro costarricenses.

En cuanto a su lugar de formación, todas llevaron el curso de certificación en DONA International, en la Universidad Estatal a Distancia (UNED), Costa Rica, el cual tuvo una duración de cinco días presenciales, mas no todas fueron certificadas. Tras cumplir con lo mencionado, los requisitos para la certificación consistieron en lecturas, ensayos, prácticas y formularios que debían llenar; sin embargo, al final solamente tres de las doulas entrevistadas completaron los requisitos de DONA y se certificaron.

En relación con el tiempo de laborar como doulas, la que cuenta con menos años de experiencia tiene tres años, mientras que la más experimentada se ha desempeñado durante 18 años, cuyos primeros ocho años fueron de conocimiento empírico, en otras palabras, realizó labores de doula sin tener preparación académica tal y como la misma refiere: "cuando yo ya hice ese curso ya tenía 8 años de ser doula, fue como no sé, la verdad lo hice para tener un papel que nunca ningún papá pidió” (entrevista realizada a doula \#7, 21 de agosto del 2014).

Además de sus labores como doulas, todas las entrevistadas poseen profesiones o prácticas alternativas que en muchos casos complementan con su desempeño como doulas, ya que para ellas representa una atención integral que les permite brindar un servicio más completo a la familia gestante, en áreas como las siguientes: psicología, educación prenatal, antropología, instrucción de yoga prenatal, técnica dental, comunicadora social, consejeras de lactancia, traductora, educadora de francés, asistente de partera y terapeuta holística (masaje, aromaterapia, musicoterapia), aunque no representa un requisito al brindar sus servicios. 


\section{Revista Electrónica Enfermeria Actual en costa Rica}

\section{Desempeño de las doulas en el periodo gestacional}

Mediante la información recolectada se verificó que no existe formación académica dentro fuera del país para las doulas en esta etapa, ya que la institución DONA internacional se especializa en la capacitación para el parto y para el post parto, tal como lo menciona la doula \#2,

Yo como doula de parto mi papel es en el parto, no existe la doula preparto, o sea la labor de la doula es... por ejemplo otras doulas son preparadoras de parto, son educadoras prenatales, y eso lo vinculan luego con el trabajo como doulas, pero en realidad... no existe doula preparto (entrevista realizada a la doula \# 28 de enero del 2014).

En las entrevistas y observaciones se identifica que, en Costa Rica, las Doulas improvisan en cada una de sus intervenciones, ya que se desempeñan de acuerdo con las necesidades de las usuarias, es decir, el enfoque de cada consulta va a depender de las necesidades que la doula identifique, por lo que ninguna de las intervenciones es igual a la anterior, tal como se menciona en la siguiente cita:

El éxito de una doula es poder tener el discernimiento y poder tener una inteligencia para poder captar como es esa mamá para poder ver la forma en que usted le habla, que la toca, que le sugiere, es completamente diferente (entrevista realizada a la doula \# 7, 21 de agosto del 2014)

En términos generales, los temas y actividades durante las intervenciones de las doula, en la mayoría de las ocasiones involucran interpretación de conceptos médicos, aclaración de dudas, preparación para el parto, acompañamiento, elaboración del plan de parto, pero sin lugar a duda los temas predominantes en estas intervenciones son la práctica de técnicas de relajación y diferentes técnicas de respiración.

\section{Desempeño durante el parto}

Al ser la doula formada propiamente para el acompañamiento durante esta etapa, en este estadio se enfoca la mayor parte de su desempeño. Al respecto, la doula \#2 refiere que la persona que se prepara académicamente para ser doula de parto, únicamente debe acompañar a la familia en esta etapa; sin embargo, en Costa Rica las doulas de parto realizan su acompañamiento en las tres etapas, tanto en el periodo gestacional como en el postparto, aunque la esencia de su trabajo se lleve propiamente durante el parto. Aunado a tales funciones, también deben acompañar y guiar el actuar de la pareja o acompañante.

Por otra parte, la doula \# 5 describe a sus colegas como guardianas de la oxitocina, ya que velan el proceso, velan porque la mujer tenga la confianza para tomar decisiones sobre su cuerpo y sobre su bebé, además cuidan que se esté trabajando toda la comunicación con los proveedores de salud, llámese a estos personal de enfermería y médicos tratantes, mas su labor no radica en interferir, ni dar opiniones.

No obstante, a pesar de que algunas en su actuar no interfieren directamente, tratan de persuadir a la madre o al padre en cuanto a recordarles lo que originalmente ellos esperaban de su parto, de modo que cuestionen al médico o enfermera(o) obstetra sobre su actuar, por ejemplo que se pregunten el porqué de la colocación de suero, de realizar la cesárea y de no realizar el parto en el cuarto, en lugar de la sala de partos, etc. 
Por otra parte, según lo expresado por las doulas entrevistadas, su desempeño dependerá del lugar donde realicen el acompañamiento, ya sea en casa o en hospitales públicos o privados, puesto que en la casa, además de desempeñar el rol de doula, ejecuta otro tipo de labores, como "cocinar una comidita sencilla, medio acomodar la casa, incluso, hasta lavar la ropa; la doula antes de llegar al lugar le puede preguntar si necesita algo del supermercado" (entrevista realizada a la Doula \# 3, 23 de Enero del 2014)

Por otro lado, en el ámbito hospitalario, la no apertura principalmente en hospitales públicos, les imposibilita desempeñarse en su rol, pese a que, como menciona una ellas "el trabajo de una Doula se ve como realmente es en los hospitales, porque los partos en casa son un poco más como informales, en los hospitales se ve más porque hay enfermeras, hay todo un equipo con bastantes roles". (entrevista realizada a la doula \# 6 , el 23 de Julio del 2014).

Al ser entrevistadas, la mayoría demuestra mucha seguridad al desempeñarse en el parto y veracidad en su discurso; sin embargo, durante las entrevistas fue posible encontrar que casi en su totalidad realizan otras labores no consideradas dentro de sus funciones, como por ejemplo fungir como asistentes de la partera al momento del parto. Una de ellas argumenta que, según lo estipula DONA International, asistir a una partera no corresponde al trabajo de una doula según el perfil de dicha institución.

\section{Desempeño durante el postparto}

En cuanto al desempeño en el postparto, al igual que en las demás etapas, brindan acompañamiento y asesoramiento tanto a la madre como a su familia y cada una de las consultas que realizan en esta etapa tendrá un enfoque diferente, de acuerdo con las necesidades de esta familia. Respecto de lo anterior, una de ellas aclara:

Vemos mucho lo que pasó en el parto, si fue cesárea, si fue parto, traslado...cómo se sienten al respecto y, mucho, la lactancia. Vemos cómo está la lactancia, también vemos cómo está la mamá de ánimo, que es muy importante también dependiendo de cómo fue el parto, y cómo está la mamá, quien la está ayudando y quien la está asistiendo(entrevista realizada a la doula \# 6).

Del mismo modo, la anterior doula comenta que, como parte de sus labores, ellas ayudan a colocar una faja a nivel abdominal para ayudar a las madres en su recuperación, a lo que se agrega "baños con hierbas especiales, dependiendo de cómo se sienta la mamá, hacemos baños con agua de romero, si necesitan lo que sea de afuera les traemos lo que sea, cuidar mucho que no haya depresión postparto, y así" (entrevista realizada a la doula \# 6, el 23 de julio del 2014). Sin embargo, en cuanto a los signos de depresión postparto, no están capacitadas para intervenir, por ende, ante este acontecimiento su papel primordial consiste en mencionar la situación a los profesionales competentes para resolverlo.

En esta etapa, entre las labores de la doula, sobresale la identificación de sus sentimientos con respecto a lo vivido en el momento del parto, la Doula \# 5 refiere que lo que ellas tratan es que "de cada historia se rescate todo lo positivo, porque al final esos son los recuerdos que van a quedar para toda la vida entonces la idea es ayudar a que sea un buen recuerdo" (entrevista realizada a la doula \# 5, 11 de Diciembre del 2013). 
Por otro lado, algunas doulas realizan prácticas ortodoxas en las que utilizan la placenta como elemento simbólico con el que preparan batidos para que la mamá los ingiera y realizan impresiones en papel, construyendo pinturas en forma de árbol, lo cual es confirmado por la doula \#2 quien comenta: "Hay otras doulas que hacen batidos, entonces les hacen batidos a las mamás poderosísimos, y luego con las placentas hacen batidos y se los toman".

\section{DISCUSIÓN}

La obstetricia ha estado presente en la historia de la humanidad desde hace muchos años, motivo por el que es fundamental estudiar los aspectos que involucran la práctica de esta disciplina en otras profesiones para entender los procesos que atraviesan las mujeres en estado de gestación y sus familias, con el fin de brindarles herramientas con las que tomen el control sobre su cuerpo y maternidad, máxime considerando que, según Cabero, Saldívar y Cabrillo (2007), "la obstetricia realiza procedimientos preventivos, diagnósticos y terapéuticos dirigidos a garantizar el bienestar específico de salud general de la madre y su criatura.” (p. 3).

Por lo mencionado, actualmente se ha trabajado mucho sobre el tema del parto respetado, el cual invita a concienciar a los profesionales de obstetricia y el personal de salud en general que trabajan con mujeres embarazadas acerca de la relevancia de promover ambientes aptos para las mujeres, dentro de lo que se enfatiza la toma de control por parte de estas en el proceso de parto, de modo que se convierten en protagonistas e involucran a sus familias. En cuanto a lo anterior, respecto del parto respetado y con base en la Declaración de Ceará en el año 2000, Arnau y otros (2012) mencionan que "la aplicación de la humanización en los cuidados que se proveen al comienzo de la vida humana será determinante y definitiva para las sociedades futuras” (p. 231).

Las acciones de un parto respetado ofrecen a la mujer, su bebé y familia atención de calidad, un equipo de salud capacitado, especializado en obstetricia, factor que disminuye el riesgo de complicaciones o eventos adversos y aumenta la seguridad de la madre y el infante.

Las doulas son mujeres que trabajan acompañando a mujeres en pro de un parto digno, tal como ellas mismas lo definen. Por ejemplo, en Jujuy, un grupo de mujeres que se denominan "Doulas comunitarias" comenzó a trabajar en la necesidad de humanizar el parto. A ellas también se les conoce también como "auxiliares de parto" entrenadas para apoyar a la mujer mediante una atención focalizada en la que brindan palabras cariñosas y positivas. (Yuyuyalmomento.com).

En relación con el tema, Funcher, Kennell y Stein (2014) mencionan que la doula es una mujer con experiencia en el parto que ofrece apoyo continuo tanto físico como emocional al momento durante y después del parto, tanto a la madre como a su pareja, a quienes se dedica al 100\%. Su principal actuar se centra en las necesidades emocionales de la mujer, mas no se involucra en el tratamiento y asuntos del personal de salud (p.1487).

Como se evidenció en los resultados, el acompañamiento de las doulas en Costa Rica ha tenido una lenta introducción y difícil aceptación, ya que no existe apertura por parte de entes gubernamentales, motivo que las obliga a desempeñar sus labores en el ámbito privado, a diferencia de otras naciones europeas y norteamericanas, donde su aceptación es tal que laboran como una funcionaria más en hospitales tanto públicos como privados. 
La DONA internacional, una de las instituciones donde se capacitan, es una organización sin fines de lucro cuyo objetivo es capacitar mujeres para el acompañamiento durante el parto y postparto, con el fin de proporcionar la más alta calidad en las normas para el acompañamiento durante el nacimiento y el apoyo postparto a las parturientas y sus familias. Esta institución provee educación continua y proporciona un enlace de comunicación entre las doulas, las familias y la comunidad médica del parto (DONA, 2012, p.1).

A pesar de que el curso para la certificación de doulas se ha impartido en Costa Rica, ellas deben cumplir los requisitos para certificarse en DONA International de Estados Unidos, pues no existe una organización que las regule o prepare adecuadamente para realizar dicha práctica en Costa Rica. Como norma, tal entidad.

Esta les prohíbe la prescripción de medicamentos o algún tipo tratamiento, por tanto, tan solo deben aconsejar a la madre a consultar con algún proveedor de servicios de salud. Cabe resaltar en este punto, que dicha formación no es académica profesional, por lo que su desempeño corresponde a un oficio y no a un ejercicio profesional, de modo que, básicamente, solo deben apoyar, informar y ser mediadoras. Si la madre o su pareja presentaran necesidades más allá del alcance de la formación de la doula, esta debe referirla a los recursos apropiados (DONA, 2008).

En Costa Rica, su quehacer es establecido por las normas de DONA; sin embargo, al no tener un ente regulador y no encontrarse todas certificadas en esta identidad, poseen más libertad en el actuar, por lo que lo complementan con conocimientos agregados, es decir, con sus prácticas u oficios alternativos a los que, en algunos casos, se agrega las labores de asistente de partera, incluso, de valoración psicológica para identificar si la usuaria presenta o no algún tipo de depresión post parto. Lo anterior se convierte en un primer diagnóstico de la situación, el cual incumple con las normas propuestas por DONA, aun cuando se establece un perfil diferente del concepto tradicional de doula.

Por otra parte, se destaca la importancia de que exista una persona, en este caso mujer, encargada de acompañar a la madre e incluso a su pareja, de brindar ese apoyo físico y emocional que muchas veces los proveedores de servicios de salud no pueden realizar, lo cual funge como una ayuda para la enfermería obstétrica, ya que la carga laboral y la institucionalización de los servicios que se ofrece a las madres no permite a los profesionales en obstetricia brindar ese apoyo de la forma en que la madre y su familia lo necesitan, lo cual concuerda con Funcher, Kennell y Stein (2014), quienes agregan que,

en la actualidad, las exigencias en materia de trabajo y enfermeras obstetras son tan grandes que pueden gastar menos que el $10 \%$ de su tiempo proporcionando servicios de apoyo. Al añadir soporte de una doula continua, los investigadores encontraron notables efectos perinatales positivos, hubo reducciones en los partos por cesárea en un $50 \%$, en el uso de fórceps por $40 \%$, en la aplicación de analgesia epidural por $60 \%$, y una disminución del $25 \%$ en la longitud de trabajo (Funcher, Kennell y Stein, 2014, p.1489).

Según la evidencia de la información recolectada, el acompañamiento y apoyo durante el periodo postparto es una necesidad que se debe solventar en las madres, motivo por el que las doulas cumplen un papel esencial en este periodo, ya que en muchos casos las madres salen del hospital después del parto con muchas dudas y miedos y al llegar a sus casas, a pesar de que tienen el apoyo de su pareja o familiares, se sienten solas, confundidas, con 
sentimientos que no logran expresar adecuadamente en muchos casos, situación que puede afectar el vínculo de la madre con su nuevo bebé, por tanto, según Otero y Prieto (2013) "en el postparto inmediato, la doula fundamentalmente velará por el establecimiento de la lactancia y porque las condiciones sean las óptimas de cara al vínculo entre madre y bebé” (p.30).

En resumen, el objetivo de la enfermera obstetra es garantizar un resultado seguro, mientras que el de la doula es asegurar que la mujer se sienta segura y confidente (Ballen y Fulcher, 2006, p.305), por lo que sería valioso el acompañamiento de una doula en la enfermería obstétrica; sin embargo, un requisito esencial es que esté inscrita en alguna organización que la respalde y rija su actuar en cuanto a las normas y código ético.

En la actualidad el papel de la Doula no ha sido ampliamente adoptado en la atención obstétrica debido a la falta de comprensión de su papel, lo cual puede relacionarse con el desconocimiento de su desempeño o el rechazo del comportamiento que algunas doulas presentan cuando no se apegan a las normas establecidas (Ballen y Fulcher, 2006, p.304).

Finalmente, el papel de las doulas en Costa Rica se encuentra directamente relacionado con las prácticas y profesiones alternativas con las que complementan su desempeño, al punto de que modifican su desempeño tradicional y crean un modelo de doula original, basado en los principios establecidos por DONA International pero sin la necesidad de cumplir con las normas y doctrinas de ese ente, ya que a nivel nacional no se cuenta con una institución que regule su práctica por medio de un código de ética y que las caracterice a nivel profesional, carencia que propicia situaciones o prácticas que no corresponden a su perfil.

\section{CONCLUSIONES}

- Todas las doulas en Costa Rica se capacitan en prácticas alternativas para complementar su labor, aspecto que para ellas significa la posibilidad de mejorar la atención que brindan a sus clientas. Algunas de estas prácticas alternativas son yoga, masaje, técnicas de relajación (control de la respiración, aromaterapia, musicoterapia), terapias holísticas, entre otras. Las doulas se mantienen en una continua actualización personal de conocimientos en temas como lactancia materna y educación prenatal, sin embargo, cabe destacar que capacitarse y brindar estos servicios a las madres no forma parte del perfil de la doula establecido por DONA International, ya que su labor se basa únicamente en brindar apoyo y acompañamiento.

- Es imprescindible mencionar que en la mayoría de los casos en Costa Rica, las doulas realizan prácticas e intervenciones que no forman parte de su perfil, por consiguiente, es fundamental contar con una entidad que regule su práctica y que vele porque las doulas se apeguen a los principios bioéticos establecidos por DONA International y a su rol sin caer en prácticas indebidas.

- A partir de las observaciones y entrevistas es posible concluir que el apoyo continuo a la mujer y su familia durante todo su embarazo, parto y postparto por una persona calificada mejora la experiencia de su parto y disminuye los riesgos de un evento adverso para ella o su bebé, razón por la que se debe sensibilizar a la población de enfermeros (as) obstetras respecto de mejorar la calidad en la atención que se brinda a las mujeres 


\section{Revista Electrónica Entermeria actualen costa Rica}

embarazadas, al tiempo que se promueve el acompañamiento continuo de la mujer por parte de personal capacitado.

- Se considera pertinente incluir a nivel hospitalario la labor de la doula como un servicio complementario a enfermería obstétrica como sucede en algunos países de Europa, sin irrespetar el perfil de esta que únicamente consiste en acompañamiento y apoyo, sin caer en intervenciones propias de enfermería obstétrica, proceso normado por un ente formador y regulador en el país.

- Por otro lado, urge concienciar al personal de salud que labora con familias gestantes sobre la importancia de velar por los derechos de la mujer durante el parto, respetar los planes de parto de la madre, promover una libre posición, el derecho a mantenerse acompañada y apoyada en todo momento, mantener una información clara y transparente sobre el transcurso de su labor, entre otras aspectos que incluye un parto respetado.

\section{Agradecimientos}

Queremos agradecer a la directora de esta investigación, la Dra. Ligia Rojas Valenciano, ya que de no ser por su apoyo, confianza y dedicación, este trabajo no hubiera sido posible.

También agradecemos a las doulas que nos transmitieron sus experiencias, ya que gracias a todos sus aportes hicieron de esta investigación una fuente de enriquecimiento tanto personal como profesional, sin olvidar el interés y carisma que mostraron durante todo el proceso.

Finalmente, agradecemos a las familias que nos permitieron estar presentes en las consultas prenatales, ya que confiaron en nosotras e hicieron posible esta investigación.

\section{REFERENCIAS BIBLIOGRÁFICAS}

Arnau, J.; Martínez, M.; Nicolás, M.; Bas, E.; Morales, R.; Álvarez, L. (2012). Los conceptos del parto natural y humanizado. El caso del área 1 de salud de la región de Murcia. Revista de Antropología Iberoamericana, 7(2), 226-247. Recuperado de http://www.aibr.org/antropologia/07v02/articulos/070204.pdf

Ballen, L. y Fulcher, A. (2006). Nurses and Doulas: Complementary Roles to Provide Optimal Maternity Care. Rev JOGNN, 35(2), 304-311. Recuperado de https://health.ucsd.edu/specialties/obgyn/maternity/facilities/doula/Documents/NursesDoulas.pdf

Cabero, L., Saldívar, D., Cabrillo. (2007). Obstetricia y Medicina Materno-Fetal. Madrid: Médica Panamericana. Recuperado de

http://books.google.co.cr/books?id=AGh8rK1MmOsC\&printsec=frontcover\&hl=es\&source=gbs_ge_summ ary_r\&cad $=0 \# \mathrm{v}=$ onepage $\& \mathrm{q} \& \mathrm{f}=$ true 
Chinchilla, D. (2010). Aliadas a la hora del parto. La Nación. Recuperado de http://www.nacion.com/archivo/Aliadas-hora-parto_0_1143685672.html

DONA international (2005). We are the world's premier doula organization. Recuperado de http://www.dona.org/aboutus/index.php

DONA international (2008). Standards of Practice Birth Doula. Recuperado de http://www.dona.org/aboutus/standards_birth.php

DONA international (2012). Position Paper: The birth Doula's Contribution to Modern Maternity Care. Recuperado de http://www.dona.org/PDF/Birth\%20Position\%20Paper_rev\%200912.pdf

Funcher, A; Kennell, J. y Stein, M. (2014). Benefits of Doula present at the birth of a child. Oficial journal of the American of pediatrics, 114 (5), 1487 - 1492.

Kennell, J.; Klaus, M.; McGrath, S.; Robertson, S. y Hinkley, C. (1991) Continuous Emotional Support During Labor in a US Hospital. A Randomized Controlled Trial. JAMA The Journal of the American Medical Association, 265 (17), 2197-2201. Doi: 10.1001/jama.1991.03460170051032.

Martínez, M. (1997). Comportamiento humano, nuevos métodos de investigación. México, D.F: Editorial Trillas.

Martínez, M. (2006). La investigación cualitativa (síntesis conceptual). Revista de Investigación en Psicología. 9(1). Lima. Recopilado de: http://pepsic.bvsalud.org/scielo.php?script=sci_arttext\&pid=S160974752006000100009

Maxwell, J. (2005). Qualitative research design: An interactive Approach. California: Editorial Thousand Oaks.

Mendoza, C; Silva, L. y Alves, M. (2010). Participação do acompanhante na humanização do parto e sua relação com a equipe de saúde. Revista electrónica de Enfermagem, 12(2), 386-391.

Otero, N. y Prieto, S. (2013). La maternidad acompañada, una reflexión sobre la función de las doulas en el cuidado de las madres. España: Editorial OB STARE.

Papagni, K; Buckner, E. (2006). Doula Support and Attitudes of Intrapartum Nurses: A Qualitative Study from the Patient's Perspective. The Journal of Perinatal Education. 15(1). Recuperado de http://www.ncbi.nlm.nih.gov/pmc/articles/PMC1595283/pdf/JPE150011.pdf

Rodríguez, G., Gil, J., García, E. (1999). Metodología de la investigación cualitativa. Málaga: Ediciones Aljibe. 
Rojas, L. (2008). Elementos conceptuales y metodológicos de la investigación cualitativa. San José, Costa Rica. UCR.

Romero, F. (2009). Las doulas, identidad, cuestiones abiertas y propuestas para su desarrollo. EDUCER, 1(0), 1-23. Recuperado de http://www.craneosacral.org/INFANCIA/Doula.pdf

Uribe, D. y Viveros, L. (2009). Modelo de atención en salud “doulas”, para mejorar la calidad del cuidado perinatal en instituciones de primer nivel de atención en Bogotá. Revista cuadernos Latinoamericanos de Administración, 9(5), 95-119. Recuperado de

http://www.uelbosque.edu.co/sites/default/files/publicaciones/revistas/cuadernos_latinoamericanos_adminis tracion/volumenV_numero9_2009/modelo_atencion_salud_Doulas .pdf

Valdés, K. y Morlans, M. (2005). Aportes de las Doulas a la Obstetricia moderna. Obtenido de Revista Chilena de Ginecología y Obstetricia 70(2): http://www.scielo.cl/scielo.php?script=sci_arttext\&pid=S071775262005000200010

Valles, M. (1999). Técnicas cualitativas de investigación social: Reflexión metodológica y Práctica Profesional. México D. F.: Síntesis.

Doulas comunitarias de yuyuy. (2014). Hacia un parto humanizado.

http://jujuyalmomento.com/post/18237/dulas-comunitarias-de-jujuy-hacia-un-parto-

humanizado.htmlYuyuyalmomento.com 